\title{
Heel to Ear Measurement
}

National Cancer Institute

\section{Source}

National Cancer Institute. Heel to Ear Measurement. NCI Thesaurus. Code C89513.

Useful in assessing neonatal neuromuscular development, this is obtained when the infant is placed in a supine position and the infant's feet are grasped pulled toward the ipsilateral ears. The examiner measures the angle made by the arc from the table to the infant's heels; increased angle for age suggests hypotonia, and decreased angle for age suggests hypertonia. 